Research report

\title{
What is important in being cured from: Does discordance between physicians and patients matter? (2)
}

\author{
Koen Demyttenaere ${ }^{\mathrm{a}, *}$, Anne-Françoise Donneau ${ }^{\mathrm{b}}$, Adelin Albert ${ }^{\mathrm{b}}$, Marc Ansseau ${ }^{\mathrm{c}}$, \\ Eric Constant ${ }^{\mathrm{d}}$, Kees van Heeringen ${ }^{\mathrm{e}}$ \\ a University Psychiatric Centre, University of Leuven, Campus Gasthuisberg, Leuven, Belgium \\ ${ }^{\mathrm{b}}$ Department of Medical Informatics and Biostatistics, University of Liège, CHU Sart Tilman, Liège, Belgium \\ ${ }^{\mathrm{c}}$ Department of Psychiatry and Medical Psychology, University and CHU of Liège, CHU Sart-Tilman, Liège, Belgium \\ ${ }^{\mathrm{d}}$ Department of Psychiatry, Catholic University of Louvain, Cliniques Universitaires Saint-Luc, Brussels, Belgium \\ e University Department of Psychiatry and Medical Psychology, Unit for Suicide Research, University of Ghent Hospital, Ghent, Belgium
}

\section{A R T I C L E I N F O}

\section{Article history:}

Received 15 June 2014

Received in revised form

1 December 2014

Accepted 2 December 2014

Available online 10 December 2014

\section{Keywords:}

Depression

Antidepressants

Outcome

Expectations

\begin{abstract}
A B S T R A C T
Aims: The influence of discordance in what is important in being cured from depression on clinical outcome at 6 months, assessed with a divergence index.

Methods: 304 outpatients treated for depression by general practitioners or by psychiatrists and completing a 6-month treatment period: a divergence index (divergence between physician and patient view on what is important in being cured from depression) was calculated for each physician-patient pair. The relation between this index and outcome at 6 months was analyzed (including depressive, anxious and somatic symptom severity, positive effect, functional impairment and quality of life (psychological and social relations).

Results: Response rates (50\% improvement) were $65.9 \%$ for depressive symptomatology and $46.2 \%$ for anxious symptomatology. The subgroup with a poor physician-patient agreement (highest quartile) on expectations had a worse clinical outcome than the subgroup with an excellent physician-patient agreement (lowest quartile): differences in response rate between these groups ranged from $9 \%$ to $27 \%$; this difference reached statistical significance for 3 outcome variables (anxiety, positive effect and social relationships).

Conclusions: The study shows that outcomes with standard antidepressant drugs are still suboptimal and that discordance between what patients' and physicians' consider important in the definition of cure from depression significantly influences clinical outcomes at 6 months.
\end{abstract}

(c) 2014 Elsevier B.V. All rights reserved.

\section{Introduction}

A meta-regression analysis showed that response rates are $53.8 \%$ for a typical antidepressant and $37.3 \%$ for placebo suggesting that about two thirds of antidepressant efficacy is due to a non-specific placebo effect (Papakostas and Fava, 2009). But again, important differences were found between observer-rated and self-rated outcomes. Using non-disease specific scales, it has indeed been reported that the percentage of patients with a 'global improvement' during antidepressant treatment was 10\% higher in the physician's assessment than in the patient's assessment (Demyttenaere et al., 2009a, 2009b). Moreover, in a group of patients being in (observer-rated) remission (i.e. a HDRS - Hamilton Depression Rating Scale $-\leq 7$ ), only 55111\% considered themselves in remission (not defined, but

\footnotetext{
* Corresponding author.

E-mail address: koen.demyttenaere@med.kuleuven.be (K. Demyttenaere).
}

based on personal conceptualization of remission) (Hamilton, 1960; Zimmerman et al., 2012). Interestingly, patients in observer-rated remission who also considered themselves in remission had higher scores on positive mental health and had significantly less functional impairment than patients with only observer-rated remission (Zimmerman et al., 2012).

The reasons for these clinically important discrepancies have been insufficiently investigated and they could theoretically be influenced by clinical variables, trial or design features, patient attitudes and behaviors, and doctor-patient relationship. For example, it has been shown that a higher baseline discrepancy between scores derived from an observer-scale and a self-rating scale leads to a poorer outcome, is generally observed in patients with a comorbid personality disorder and positively correlates with anxiety levels (Rane et al., 2010). Factors related to patient expectations from antidepressant have also been shown to influence outcome. Response rates in patients treated with an antidepressant were found to be $65.4 \%$ in drug-drug studies, $57.7 \%$ in 
drug-drug-placebo studies and $51.7 \%$ in drug-placebo studies while in patients treated with placebo, response rates were $44.6 \%$ in drug-drug-placebo trials and $34.3 \%$ in drug-placebo trials (Sinyor et al., 2010). This is further illustrated by a study showing higher HDRS scores immediately postrandomization (moving to placebo or staying on fluoxetine): this increase in HDRS scores was found as well in patients staying on fluoxetine as in patients moving to placebo (Rutherford et al., 2014). This suggests that treatment changes influence paitents' expectations of improvement, which, in turn, affect their depression symptom severity. These data suggest that the chance of being on active treatment (through physician and patient expectations) is an important predictor of outcome. Another study investigating the influence of baseline attitude to taking (antidepressant) medication showed that patients with a baseline positive/neutral/negative attitude towards antidepressants presented a placebo response rate of $46 \%, 37 \%$ and $30 \%$ and an antidepressant response rate of $67 \%, 60 \%$ and $56 \%$, respectively, again suggesting that baseline expectations and attitudes predict outcome (Demyttenaere et al., 2011). The NIMH-funded study on the efficacy of hypericum perforatum did not find any significant difference in response rates between the hypericum arm, the sertraline arm and the placebo arm but a reanalysis taking into account patient's guess of which treatment they got showed important differences in outcome: patients guessing they were on sertraline had a $56 \%$ response rate, patients guessing they were on hypericum had a $68 \%$ response rate while patients guessing they were on placebo had a $24 \%$ response rate (Hypericum Depression Trial Study Group, 2002; Chen et al., 2011). Further, the attitude and behavior of the prescribing psychiatrists are also important: the psychiatrist is not only a provider of treatment but also acts as a mean of treatment. Indeed, the proportion of variability in outcomes was shown to be due less to the antidepressant treatment received (imipramine or placebo) than to the psychiatrist administering the treatment (McKay et al., 2006). Interestingly, the psychiatrist effects were not as dramatic for the observer-rated measure than for the self-report measure $(6.7 \%$ and $9.1 \%$ of the variance, respectively).

We previously showed that what physicians and patients consider to be important in being cured from depression is different, and the present paper investigates whether these differences influence outcome in depressed patients treated with antidepressants.

\section{Methods}

Ethics statement, patient recruitment and data collection were described in the previous paper (Demyttenaere et al., submitted).

\subsection{Treatment and clinical outcome}

The protocol stipulated that the treatment was entirely left to the discretion of the physician: one antidepressant was prescribed to $92.5 \%$ of the patients and two antidepressants were prescribed to $7.1 \%$ of the patients (no antidepressant was prescribed to $0.2 \%$ of the patients and 43 different antidepressants were prescribed to $0.2 \%$ of the patients). In $45.2 \%$ of the patients, at least one modification in the antidepressant treatment was observed during the 6-month study period.

The clinical status of the patient was assessed with the following mentioned self-rating scales: the Patient Health QuestionnaireDepression (PHQ-depression; depressive symptoms), Hospital Anxiety and Depression Scale-anxiety (HADS-anxiety; anxious symptoms), Patient Health Questionnaire-Somatic Symptoms severity (PHQ-somatic; somatic symptoms), the Positive And Negative Affect Schedule-Positive effect subscale (PANAS-positive effect), the
Sheehan Disability Scale (SDS; functional impairment), and the Abbreviated World Health Organization QoL (WHOQOL-BREF; quality of life-psychological and social relationships), at baseline and after 6 months of treatment (Kroenke et al., 2001, 2002; Zigmond and Snaith, 1983; Watson et al., 1988; Sheehan et al., 1996; WHO-QOL group, 1998).

\subsection{Statistical analysis}

Discordance index based on the top 10 DEsCRIBE ${ }^{\mathrm{TM}}$ items of the patient.

The discordance between what patients and physicians consider important in defining cure from depression was based on their respective baseline scores on the DEsCRIBE ${ }^{\mathrm{TM}}$ questionnaire, where a ranking (importance for being cured from disorder) was given to depressive, anxious and somatic symptoms, positive effect, disability and quality of life items (Demyttenaere et al., submitted). A discordance index (DI) was constructed to evaluate the baseline agreement between physician and patient on the definition of being cured from depression. The discordance index was calculated for each possible pair (physician-patient) by using the differences between the item scores given by the physician and his/her patient. The items considered in the construction of the discordance index were the $10 \mathrm{DEsCRIBE}^{\mathrm{TM}}$ items classified in first position of importance by the patients in the definition of being cure from depression. To correct for the fact that patients always gave higher scores than physicians, individual patient's scores were standardized by subtracting the corresponding mean of each item. The same correction was applied to the individual physician's scores. The absolute difference between the standardized score of the patient and the standardized score of the physician was calculated for each of the 10 selected items. The discordance index was then defined as the average of the 10 absolute differences. DI scores range between 0 and 5 , where high values indicate strong divergence and low values strong agreement between physician and patient about the definition of being cured from depression

$\mathrm{DI}=\sum_{i=1}^{10} \frac{\mid(\text { score item } i-\text { mean item } i) \text { phys }-(\text { score item } i-\text { mean item } i) \text { pat } \mid}{10}$

The mean DI was $1.1 \pm 0.57$ and the median 0.99. Based on their DI value, patients were classified into 4 groups (according to the quartiles): excellent agreement with physician ( $\mathrm{DI}<0.71$ ), rather good agreement $(0.71 \leq \mathrm{DI}<0.99)$, rather poor agreement $(0.99 \leq \mathrm{DI}<1.47)$, and poor agreement (DI $\geq 1.47)$.

Results were summarized as mean and standard deviation (SD) for quantitative variables and scores; counts and proportions (\%) were used for categorical variables. The correlation coefficient was computed to measure the association between two quantitative variables. Mean values were compared by one-way analysis of variance (ANOVA). Proportions were compared by the chi-squared test for contingency tables. The comparison of the clinical score at 6 months according to DI-categories was made by ordinal logistic regression. A Cochran-Armitage test for trend was used to compare the 6-month response rates between the DI groups. Results were considered significant at the $5 \%$ critical level $(P<0.05)$. Calculations were always done on the maximum number of data available. All statistical calculations were performed by using SAS (version 9.3 for Windows) and S-PLUS (version 8.1) packages.

\section{Results}

Of the 426 patients analyzed in the study, 304 (71.3\%) completed the 6-month follow-up. Clinical status (responses and 
50\%-improvement rates) for the different clinical scales used to assess the outcome are given in Table 1. The PHQ-depression scores at 6 months showed that $11 \%$ of the patients were still suffering from major depression, $65.9 \%$ were responders (50\% improvement) and only $49 \%$ were in remission (PHQ depression $\leq 4$ ). The HADSanxiety scores at 6 months showed that $24.4 \%$ of the patients were still probable cases for an anxiety disorder and that $46.2 \%$ were responders ( $50 \%$ improvement). Treatment changes between baseline and 6 months follow-up were found in $45.2 \%$ of the patients, and increasing the dose was the most frequently found strategy (21.5\%) followed by switching (9.2\%). The largest improvement, i.e. response rates, was found for depressive symptoms, functional impairment and psychological aspects of quality of life $(65.9 \%$, $54.9 \%$ and $52.7 \%$, respectively) and the smallest improvement was found for social relationships in quality of life, somatic symptoms and anxiety symptoms (35.7\%, $46.2 \%$ and $46.2 \%$, respectively).

The discordance index based on the top $10 \mathrm{DEsCRIBE}^{\mathrm{TM}}$ items of the patient, reflecting how far the physician reported important items in defining cure from depression were from the patient reported ones, was negatively correlated with the age of the patient $(r=-0.10$; $P=0.03$ ). The discordance index was also significantly higher in dropouts than in completers $(1.20 \pm 0.55$ versus $1.10 \pm 0.58 ; P=0.03)$, especially in the subgroup of drop-outs due to patient's refusal to continue the medication $(1.38 \pm 0.54)$. Finally, the discordance index was significantly correlated with the length of the current depressive episode (weeks) $(r=0.13 ; P=0.006)$ but not with the number of previous episodes.

The relation between the baseline discordance index and clinical outcome was tested in two ways: excellent, rather good/ good, rather poor/weak and poor/poor baseline agreement versus 6 -month mean score and versus 6-month response rates $(50 \%$ improvement, i.e. a decrease of minimum 50\% from baseline for the scales assessing depression, anxiety, somatic symptoms, functional impairment) or an increase of minimum $50 \%$ from baseline for the scores on positive effect-quality of life) for the different scales. When looking at the mean scores at 6 months, a greater discordance between physician and patient resulted in a numerically worse score on all scales but this reached statistical significance only for somatic symptoms (and with a trend for depressive symptomatology, anxious symptomatology and positive effect) (Table 2 and Fig. 1a). Response rates on the depression questionnaire for excellent, rather good, rather poor and poor agreement were $72 \%, 62 \%, 66 \%$ and $63 \%$, respectively $(P=0.68)$ and remission rates on the same questionnaire (PHQ-depressive $\leq 4$ ) were $56 \%, 48 \%, 44 \%$ and $46 \%$, respectively $(P=0.49)$. When looking at response rates at 6 months, a poorer agreement resulted in a numerically lower response rate on all scales but this reached statistical significance only for anxiety symptoms, positive effect and social relationships in quality of life (Table 3 and Fig. 1b).

\section{Discussion}

The current study again underscores the suboptimal treatment in patients with a major depressive episode. After 6 months, response (50\% improvement) in depressive symptomatology is achieved in only $65.9 \%$ of the patients, and remission only in $49 \%$ of the patients. The outcome is even worse when looking at the accompanying anxiety symptoms where response is achieved in only $46.2 \%$ of the patients. These figures are in the same range as those found in other large studies. The FINDER study showed a 6month remission rate of $50 \%$ for depression and also a lower remission rate $(40 \%)$ for anxiety disorders; STAR*D found a $14-$ week and a 1-year (with up to 4 treatment steps) remission rate of $37 \%$ and in $67 \%$ of patients, respectively (Demyttenaere et al., 2009a, 2009b; Rush et al., 2006). It is worrying that after 6 months
Table 1

Clinical status at baseline and at 6 months.

\begin{tabular}{llcl}
\hline Scale & Baseline & 6 months & $\begin{array}{l}\text { 6-month response } \\
\text { rate (\%) }\end{array}$ \\
\hline PHQ9-depression & $16.5 \pm 5.8$ & $6.40 \pm 5.6$ & 65.9 \\
HADS-anxiety & $12.9 \pm 3.9$ & $7.11 \pm 4.5$ & 46.2 \\
PHQ-somatic & $9.69 \pm 4.8$ & $5.86 \pm 4.4$ & 37.6 \\
PANAS-positive & $21.1 \pm 7.1$ & $31.4 \pm 9.3$ & 47.1 \\
SDS-total & $19.2 \pm 6.8$ & $10.0 \pm 9.0$ & 52.8 \\
WHOQOL-BREF psychological & $35.6 \pm 16.3$ & $58.6 \pm 20.6$ & 52.7 \\
$\begin{array}{l}\text { WHOQOL-BREF social } \\
\text { relationships }\end{array}$ & $44.6 \pm 21.2$ & $60.0 \pm 22.2$ & 35.7 \\
\hline
\end{tabular}

of treatment, $11 \%$ of the patients still fulfill criteria for major depression and $24 \%$ are yet probable cases for an anxiety disorder. In the FINDER study, these figures were $25 \%$ and $33 \%$, respectively (Demyttenaere et al., 2009a,2009b). Since remission is the goal of antidepressant treatment, compliance with guidelines (on the physician side) is quite poor; although remission (for depression) was reached in only $49 \%$, changes in the treatment regimen were noticed in merely $45 \%$ of patients. However, these figures are higher than what was found in a very large naturalistic database where therapy change rates during treatment with third-, secondand first-generation antidepressants was 19\%, 21\% and $43 \%$, respectively (Sheehan et al., 2008).

A discordance index was calculated to assess how much the physician reported important items in defining cure from depression were from the patient reported ones at baseline. A lower discordance was found in older patients and a higher one in patients with a longer duration of the current episode. The baseline discordance was also higher in drop-outs than in completers and especially in the subgroup of drop-outs due to the patient's refusal to continue the medication, possibly suggesting that a high concordance of expectations is related to a better adherence to treatment.

The relation between the baseline discordance and clinical improvement during 6 months of treatment was also investigated. Interestingly there was a consistent trend for all outcome variables (at 6 months) that a weak discordance between physicians and patients resulted in a better outcome, although often only numerically. For example, the difference in response rates between the highest and lowest quartiles of the index (i.e. excellent versus poor agreement) amounted $9 \%$ for depression, $22 \%$ for anxiety, $6 \%$ for somatic symptoms, $27 \%$ for positive affect, $15 \%$ for functional impairment, $13 \%$ for the psychological aspects of quality of life, and $15 \%$ for the social relationships aspects of quality of life; these differences reach statistical significance for anxiety, positive affect and the social relationships aspects of quality of life. To put these figures into perspective, it is important to notice that in randomized clinical trials, the difference in response rates between antidepressants and placebo is $15.5 \%$. Further, the difference in response rates between patients with a positive attitude towards taking an antidepressant and patients with a negative attitude towards taking an antidepressant reached $16 \%$ in patients on placebo and $11 \%$ in patients on an antidepressant (Demyttenaere et al., 2011). Anyhow, these differences taking into account expectations and attitudes are much larger than differences between different classes of antidepressant drugs: e.g. it was shown that the difference in response rates between venlafaxine and SSRIs was $4.3 \%$ in 8 -week randomized clinical trials and that the difference in remission rates in a 6-month open-label trial between venlafaxine and SSRIs was only 3.5\% (Papakostas et al., 2007; Thase et al., 2011).

To the best of our knowledge, this is the first paper investigating whether the discordance between what physician or patients 
Table 2

Baseline discordance index and clinical status at 6 months (scores on different psychometric tests).

\begin{tabular}{|c|c|c|c|c|c|}
\hline \multirow[t]{2}{*}{ Scale } & \multicolumn{4}{|c|}{ Baseline agreement between physician and patient } & \multirow[t]{2}{*}{$P$-value* } \\
\hline & Excellent & Rather good & Rather poor & Poor & \\
\hline PHQ9-depression & $5.7 \pm 5.3$ & $6.2 \pm 5.3$ & $6.8 \pm 5.9$ & $7.1 \pm 6.1$ & 0.09 \\
\hline HADS-anxiety & $6.0 \pm 4.1$ & $7.7 \pm 4.0$ & $7.2 \pm 4.9$ & $7.6 \pm 4.9$ & 0.07 \\
\hline PHQ-somatic & $5.2 \pm 3.9$ & $5.7 \pm 3.9$ & $6.1 \pm 5.3$ & $6.8 \pm 4.5$ & 0.04 \\
\hline PANAS-positive & $32.8 \pm 9.5$ & $31.0 \pm 8.7$ & $31.7 \pm 9.4$ & $29.7 \pm 9.5$ & 0.09 \\
\hline SDS-total & $8.9 \pm 8.9$ & $10.1 \pm 8.5$ & $9.4 \pm 8.8$ & $11.8 \pm 10.0$ & 0.11 \\
\hline WHOQOL-BREF psychological & $59.5 \pm 21.1$ & $57.8 \pm 19.4$ & $59.3 \pm 21.3$ & $56.9 \pm 21.2$ & 0.58 \\
\hline WHOQOL-BREF social relations & $62.3 \pm 21.4$ & $58.4 \pm 21.3$ & $60.5 \pm 23.9$ & $57.6 \pm 22.3$ & 0.31 \\
\hline
\end{tabular}

* Ordinal logistic regression (excellent versus poor agreement).
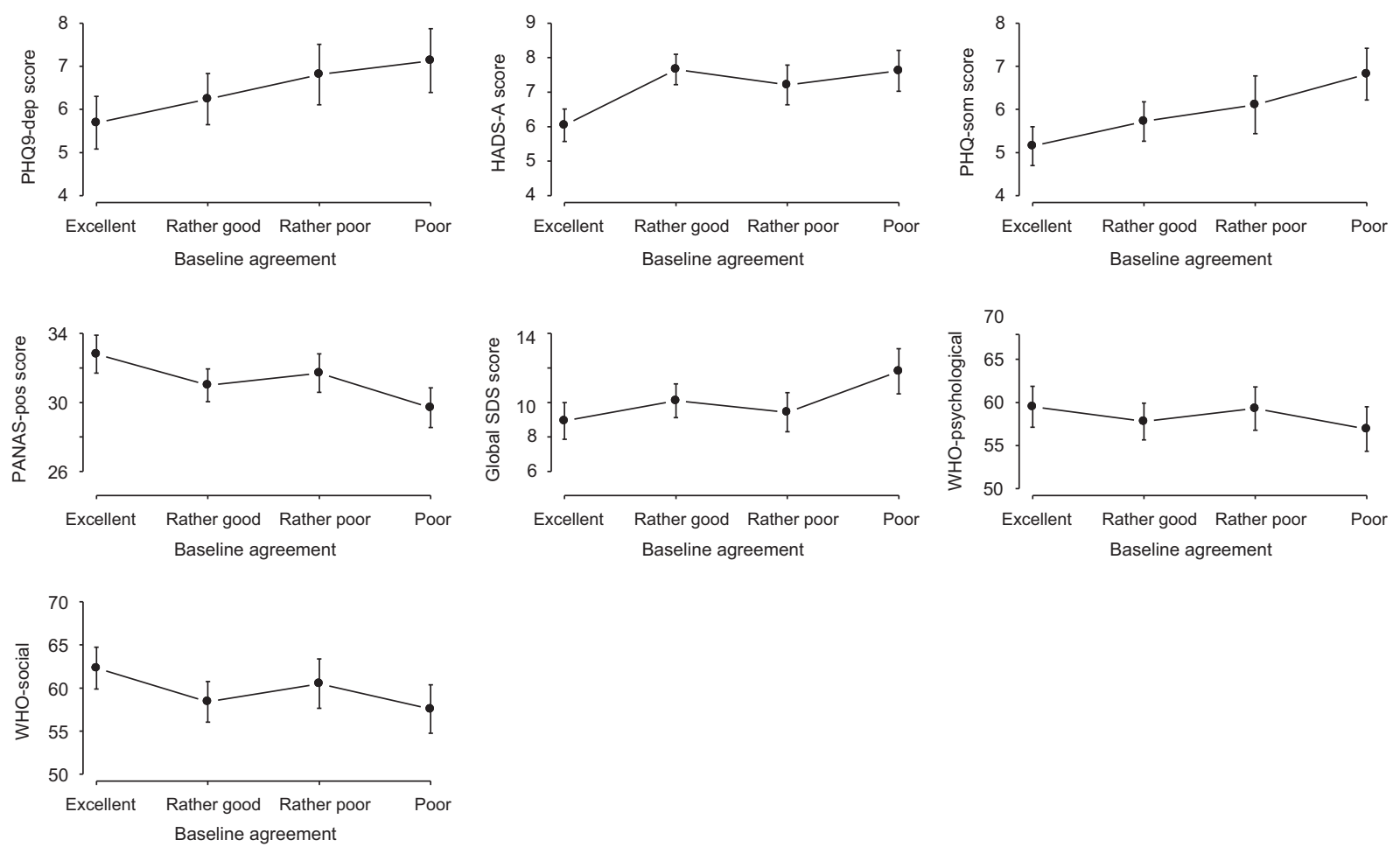

Fig. 1. Baseline agreement between physician and patient and clinical outcome: (a) clinical status at 6 months, (b) evolution from baseline to 6 months.

Table 3

Baseline discordance index and clinical status at 6 months (response rates $-50 \%$ improvement-for different psychometric tests).

\begin{tabular}{|c|c|c|c|c|c|}
\hline \multirow[t]{2}{*}{ Scale } & \multicolumn{4}{|c|}{$\begin{array}{l}\text { Baseline agreement between physician and } \\
\text { patient }\end{array}$} & \multirow[t]{2}{*}{$\begin{array}{l}P \text { - } \\
\text { value* }\end{array}$} \\
\hline & $\begin{array}{l}\text { Excellent } \\
(\%)\end{array}$ & $\begin{array}{l}\text { Rather } \\
\operatorname{good}(\%)\end{array}$ & $\begin{array}{l}\text { Rather } \\
\text { poor }(\%)\end{array}$ & $\begin{array}{l}\text { Poor } \\
(\%)\end{array}$ & \\
\hline PHQ9-depression & 72 & 62 & 66 & 63 & 0.68 \\
\hline HADS-anxiety & 62 & 41 & 44 & 40 & 0.02 \\
\hline PHQ-somatic & 42 & 34 & 38 & 36 & 0.61 \\
\hline PANAS-positive & 66 & 38 & 47 & 39 & 0.01 \\
\hline SDS-total & 63 & 52 & 56 & 48 & 0.38 \\
\hline $\begin{array}{l}\text { WHOQOL-BREF } \\
\text { psychological }\end{array}$ & 65 & 48 & 46 & 52 & 0.13 \\
\hline $\begin{array}{l}\text { WHOQOL-BREF social } \\
\text { relations }\end{array}$ & 51 & 32 & 22 & 36 & 0.02 \\
\hline
\end{tabular}

* Trend test.

report as important items in defining cure from depression influences outcome in depression. We can only speculate why this discordance significantly influences outcome (anxiety, positive affect, and social relationships in quality of life). The literature reports several studies investigating either patient or physician expectations or therapeutic alliance but not the combination of these factors. For example, a study used path analysis to show that patients' treatment expectancies significantly influenced clinical improvement but only indirectly through the patient alliance; and that therapists' global expectancies also influence clinical improvement partly directly and partly through the patient alliance (valid conclusions for the 4 different treatment arms: cognitive-behavior therapy, interpersonal therapy, imipramine+clinical management, placebo+clinical management) (Meyer et al., 2002). Another interesting paper suggested that a good communication and a good alliance are even more important for some subgroups of patients; it was for example shown that a good communication and a good alliance were important for outcome (in diabetic control) mainly in patients with a dismissing attachment style (Ciechanowski et al., 2001).

Our research protocol did not include a scale assessing doctorpatient alliance but it could well be hypothesized that more common expectations are related to a better alliance. Anyhow, a lower discordance index illustrating more common expectations from antidepressant treatment and so probably a better therapeutic alliance between a patient and a physician could augment the efficacy of the prescribed drug, have a direct effect on clinical 

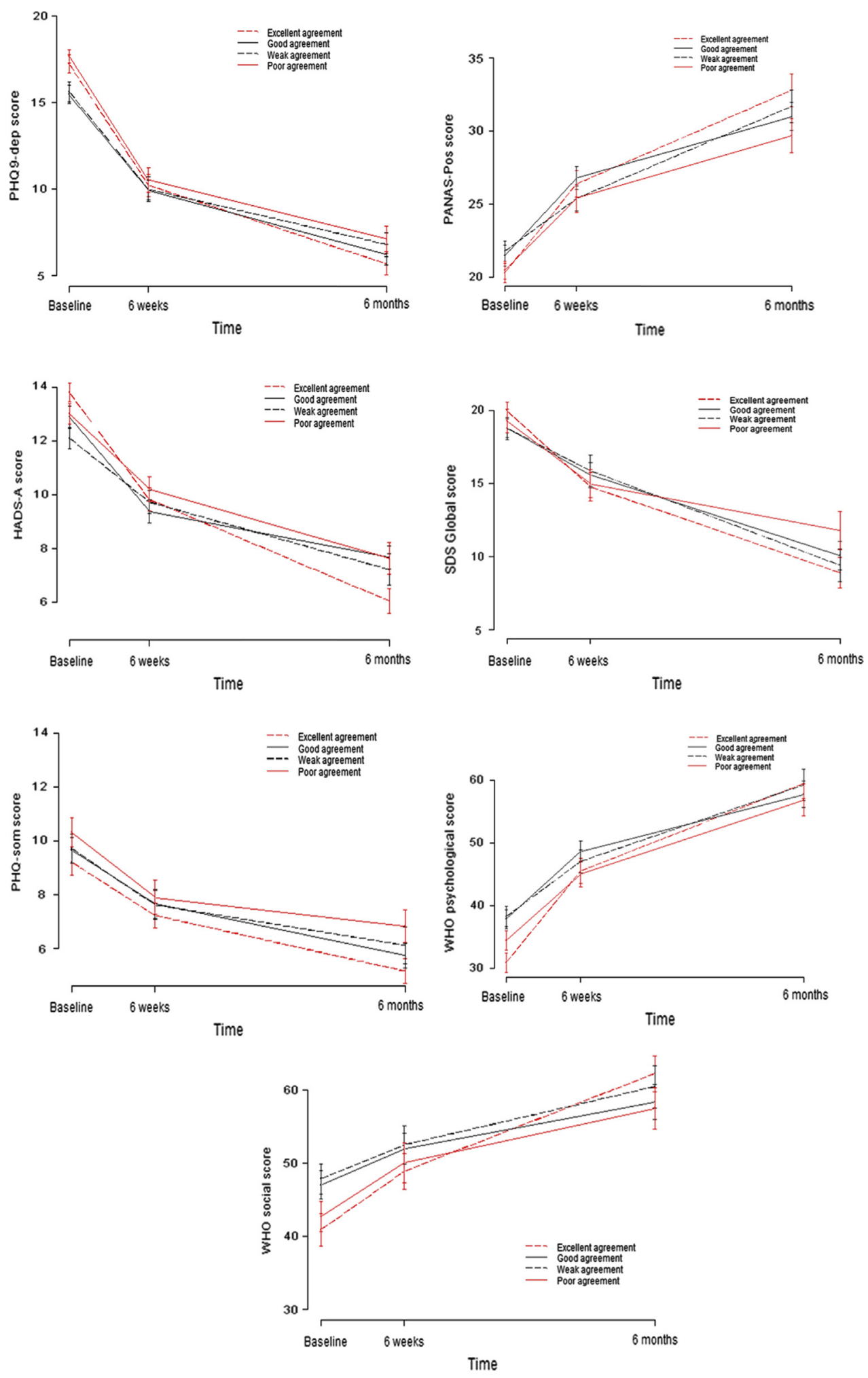

Fig. 1. (continued) 
outcome or on outcome in the presence of the prescribed drug (Zuroff and Blatt, 2006). It cannot be forgotten that about two thirds of the antidepressant effect is 'placebo' but the latter refers to the common features of care: therapeutic alliance, hope, expectation, remoralization, receipt of an explanatory system, convincing rationale, therapeutic relationship, rituals, healing context including the patient's and the physician's beliefs and representations.

In conclusion, the present study illustrates that outcomes with standard antidepressant drugs are still suboptimal and that baseline discordance between physician and patient reported important items in defining cure from depression significantly influence clinical outcomes at 6 months, mainly for anxiety, positive affect and social relationships in quality of life.

\section{Role of funding source}

Lundbeck Belgium provided the logistical support provided for performing this study; there was complete independence of researchers in the collection, analysis, and interpretation of the data and in the writing of the report, and in the decision to submit the article for publication.

\section{Conflict of interest}

All authors have completed the Unified Competing Interest form and declare that (1) A-F., D., and A.A. had financial support from Lundbeck Belgium for the statistical analysis, (2) there are no relationships that might have an interest in the submitted work in the previous 3 years, (3) their spouses, partners, or children have no financial relationships that may be relevant for the submitted work; and (4) they have no non-financial interests that may be relevant to the submitted work. Patient consent: obtained. Ethics approval: the research was approved by the Institutional Ethics Committee of the Université Catholique de Louvain (Saint-Luc Hospital). The corresponding author, also in name of the co-authors, grants worldwide license to the publisher.

\section{Acknowledgments}

All authors participated in conceiving and designing the study. Anne-Françoise Donneau and Adelin Albert analyzed the data and Koen Demyttenaere coordinated the writing of the manuscript.

\section{References}

Chen, J.A., Papakostas, G.I., Youn, S.J., Baer, L., Clain, A.J., Fava, M., Mischoulon, D. 2011. Association between patient belief regarding assigned treatment and clinical response: reanalysis of data from the Hypericum Depression Trial Study Group. J. Clin. Psychiatry 72 (12), 1669-1676.

Ciechanowski, P.S., Katon, W.J., Russo, J.E., Walker, E.A., 2001. The patient-provider relationship: attachment theory and adherence to treatment in diabetes. Am. J. Psychiatry 158, 29-35.

Demyttenaere, K., Desaiah, D., Petit, C., Croenlein, J., Brecht, S., 2009a. Patientassessed versus physician-assessed disease severity and outcome in patients with nonspecific pain associated with major depressive disorder. Prim. Care Companion J. Clin. Psychiatry 11 (1), 8-15.

Demyttenaere, K., Verhaeghen, A., Dantchev, N., Grassi, L., Montejo, A.L., Perahia, D.G. Quail, D., Reed, C., Tylee, A., Bauer, M., 2009b. Caseness for depression and anxiety in a depressed outpatient population: symptomatic outcome as a function of baseline diagnostic categories. Prim. Care Companion J. Clin. Psychiatry 11 (6), 307-315.

Demyttenaere, K., Reines, E.H., Lönn, S.L., Lader, M., 2011. Satisfaction with medication is correlated with outcome but not persistence in patients treated with placebo, escitaloprram or serotonin-norepinephrine reuptake inhibitors: a post-hoc analysis. Prim. Care Companion CNS Disord. 13 (4), http://dx.doi.org/ 10.4088/PCC.10m01080.

Demyttenaere K., Donneau A.F., Albert A., Ansseau M., Constant E., van Heeringen K. Physician and patient diverge in their expectations from antidepressant treatment (1). 2014, in press.

Hamilton, M., 1960. A rating scale for depression. J. Neurol. Neurosurg. Psychiatry 23, 56-62.

Hypericum Depression Trial Study Group, 2002. Effect of hypericum perforatum (St John's Wort) in major depressive disorder: a randomized controlled trial. JAMA 287 (14), 1807-1814.

Kroenke, K., Spitzer, R.L., Williams, J.B., 2001. The PHQ-9: validity of a brief depression severity measure. J. Gen. Intern. Med. 16, 606-613.

Kroenke, K., Spitzer, R.L., Williams, J.B.W., 2002. The PHQ-15: validity of a new measure for evaluating the severity of somatic symptoms. Psychosomat. Med. $64,258-266$.

McKay, K.M., Imel, Z.E., Wampold, B.E.J., 2006. Psychiatrist effects in the psychopharmacological treatment of depression. J. Affect. Disord. 92 (2-3), 287-290.

Meyer, B., Krupnick, J.L., Simmens, S.J., Pilkonis, P.A., Egan, M.K., Sotsky, S.M., 2002 Treatment expectancies, patient alliance, and outcome: further analyses from the National Institute of Mental Health treatment of depression collaborative research program. J. Consult. Clin. Psychol. 70 (4), 1051-1055.

Papakostas, G.I., Fava, M., 2009. Does the probability of receiving placebo influence clinical trial outcome? A meta-regression of double-blind, randomized clinical trials in MDD. Eur. Neuropsychopharmcol. 19, 34-40.

Papakostas, G.I., Thase, M.E., Fava, M., Nelson, J.C., Shelton, R.C., 2007. Are antidepressant drugs that combine serotonergic and noradrenergic mechanisms of action more effective than the selective serotonin reuptake inhibitors in treating major depressive disorder? A meta-analysis of studies of newer agents. Biol. Psychiatry 62, 1217-1227.

Rane, L.J., Fekadu, A., Wooderson, S., Poon, L., Markopoulou, K., Cleare, A.J., 2010. Discrepancy between subjective and objective severity in treatment-resistant depression: prediction of treatment outcome. J. Psychiatr. Res. 44, 1082-1087.

Rush, A.J., Trivedi, M.H., Wisniewski, S.R., Nierenberg, A.A., Stewart, J.W., Warden, D., Niederehe, G., Thase, M.E., Lavori, P.W., Lebowitz, B.D., McGrath, P.J., Rosenbaum, J.F., Sackeim, H.A., Kupfer, D.J., Luther, J., Fava, M., 2006. Acute and longer-term outcomes in depressed outpatients requiring one or several treatment steps: a STAR*D report. Am. J. Psychiatry 163, 1905-1917.

Rutherford, B.R., Wall, M.M., Glas, A., Stewart, J.W., 2014. The role of patient expectancy in placebo and nocebo effects in antidepressant trials. J. Clin. Psychiatry 75 (10), 1040-1046.

Sheehan, D.V., Harnett-Sheehan, K., Raj, B.A., 1996. The measurement of disability. Int. Clin. Psychopharmacol. 11 (Suppl. 3), S89-S95.

Sheehan, D.V., Keene, M.S., Eaddy, M., Krulewicz, S., Kraus, J.E., Carpenter, D.J., 2008 Differences in medication adherence and healthcare utilizations patterns: older versus newer antidepressant agents in patients with depression and/or anxiety disorders. CNS Drugs 22 (11), 963-973.

Sinyor, M., Levitt, A.J., Cheung, A.H., Schaffer, A., Kiss, A., Dowlati, Y., Lanctôt, K.L., 2010. Does inclusion of a placebo arm influence response to active antidepressant treatment in randomized controlled trials? Results from pooled and metaanalyses. J. Clin. Psyciatry 71 (3), 270-279.

Thase, M.E., Ninan, P.T., Musgnung, J.J., Trivedi, M.H., 2011. Remission with venlafaxine extended release or selective serotonin reuptake inhibitors in depressed patients: a randomized, open-label study (PCC.10m00979). Prim. Care Companion CNS Disord. 13 (1), http://dx.doi.org/10.488/PCC.10m00979blu.

The WHOQOL Group, 1998. Development of the World Health Organization WHOQOL-BREF quality of life assessment. Psychol. Med. 28 (3), 551-558.

Watson, D., Clark, L.A., Tellegen, A., 1988. Development and validation of brief measures of positive and negative affect: the PANAS scales. J. Personal. Soc. Psychol. 54 (6), 1063-1070.

Zigmond, A.S., Snaith, R.P., 1983. The hospital anxiety and depression scale. Acta Psychiatr. Scand. 67, 361-370.

Zimmerman, M., Martinez, J.A., Attiullah, N., Friedman, M., Toba, C., Boerescu, D.A., Rahgeb, M., 2012. Why do some depressed outpatients who are in remission according to the Hamilton Depression Rating Scale not consider themselves to be in remission? J. Clin. Psychiatry 73 (6), 790-795.

Zuroff, D.C., Blatt, S.J., 2006. The therapeutic relationship in the brief treatment of depression: contributions to clinical improvement and enhanced adaptive capacities. J. Consult. Clin. Psychol. 74 (1), 130-140. 